\title{
Neuropatía autonómica cardíaca diabética
}

\section{Diabetic cardiac autonomic neuropathy}

\author{
González $J^{1}$, Castaño $O^{2}$, Duque $M^{3}$.
}

${ }^{1}$ Médico Internista, Universidad CES. Medellín, Colombia.

${ }^{2}$ Médico Residente de cardiólogía, Universidad CES. Medellín, Colombia.

${ }^{3}$ Médico Cardiólogo y electrofisiólogo, Universidad CES. Medellín, Colombia.

Autor de correspondencia: Juanita González Arango Correo electrónico: juanitagonzalezarango@gmail.com

Fecha de recepción: 25/04/2019

Fecha de aceptación: 04/07/2019

\section{Resumen}

La neuropatía autonómica cardíaca es una complicación frecuente y subdiagnosticada de la diabetes, se asocia con una mayor mortalidad por causas cardiovasculares e incapacidad física. El tratamiento actual está basado en recomendaciones de expertos y experiencias locales, debido a la falta de estudios de alto peso epidemiológico que orienten el manejo de estos pacientes. Con esta revisión se busca generar conciencia a la comunidad médica acerca de la importancia de esta entidad, de su reconocimiento temprano, formas de diagnóstico y tratamiento disponibles con la evidencia actual.

Palabras clave: neuropatías diabéticas, diabetes mellitus, enfermedades del sistema nervioso autónomo.

\section{Abstract}

Cardiac autonomic neuropathy is a frequent and underdiagnosed complication of diabetes, associated with a high rates of mortality due to cardiovascular causes and physical disability. The current treatment is solely based on an expert's recommendation and local experiences due to the lack of evidence in patient management. With this review we seek to raise awareness in the medical community about the importance of this entity, early recognition, diagnostic methods and treatment

Keywords: diabetic neuropathies, diabetes mellitus, autonomic nervous system diseases.

\section{Introducción}

La neuropatía autonómica cardíaca es una complicación microvascular de la diabetes, que se caracteriza por una regulación anormal del sistema nervioso autónomo cardiovascular. Su prevalencia es subestimada por la falta de conocimiento de esta y poca búsqueda activa de los signos y síntomas. Es relevante el diagnóstico, ya que conlleva a mayor mortalidad y deterioro de la calidad de vida. Se debe tener en cuenta que la aparición de síntomas, que son inespecíficos, es tardía, generalmente, cuando las opciones de manejo son limitadas. De forma temprana, uno de los signos que indica el inicio de la enfermedad es la alteración en la variabilidad de la frecuencia cardíaca. En los últimos años las publicaciones sobre el tema han incrementado, lo que ha permitido un mejor entendimiento de su fisiopatología, epidemiología, manifestaciones clínicas, posibilidades diagnósticas y posible tratamiento.

\section{Revisión}

\section{Generalidades}

La diabetes ha sido llamada la epidemia del siglo XXII, con una prevalencia para el 2017, según la Federación Internacional de Diabetes, de 425 millones de personas a nivel mundial y se estima un aumento del $48 \%$ para el $2045^{(1)}$. Una de las complicaciones más comunes y, a la vez, menos diagnosticadas de la diabetes son las complicaciones cardiometabólicas, que se presentan hasta en el $60 \%$ de los pacientes. Dentro de ellas están la cardiopatía diabética y la neuropatía autonómica diabética (CAN, por sus siglas en inglés) ${ }^{(2,3)}$.

Esta complicación tiene un impacto negativo en mortalidad y morbilidad, además de ser un gran predictor de eventos cardiovasculares, como el infarto agudo de miocardio silente y muerte súbita. El riesgo de mortalidad en los pacientes con CAN es tres veces mayor que los pacientes diabéticos que no la presentan ${ }^{(4)}$, con una mortalidad estimada del $25 \%-50 \%$ a 10 años ${ }^{(5)}$. 
Dentro de los factores de riesgo para el desarrollo de esta entidad se encuentran la edad (a mayor edad, mayor prevalencia de la enfermedad), la duración de la diabetes, el control de la glucemia, la hipertensión arterial (HTA) y el desarrollo de otras complicaciones microvasculares ${ }^{(6)}$.

\section{Epidemiología}

Según la serie revisada, la prevalencia varía entre el 2,5 \%-9 0\%. Esta gran variabilidad está dada por la poca estandarización y diferentes métodos usados para el diagnóstico ${ }^{(7)}$. Los pacientes con diabetes mellitus tipo 1 (DM1) tienen una prevalencia alrededor de $30 \%$ después de 20 años del diagnóstico; y los pacientes con DM2, del $60 \%$ después de 15 años del diagnóstico ${ }^{(3)}$. En el estudio Diabetes Control and Complications Trial (DCCT) se encontraron anormalidades en la frecuencia cardíaca (marcador indirecto de CAN) según el tiempo de progresión de la diabetes: 1,6 \% DM <5 años, 6,2 \% DM 5-9 años y $12,2 \%$ DM >9 años. Esto demostró que, a mayor tiempo de la enfermedad, mayor riesgo de presentar la complicación ${ }^{(8)}$.

\section{Fisiopatología}

La fisiopatología de la enfermedad no es diferente a la de otras complicaciones microvasculares de la diabetes. La hiperglucemia junto con otros factores, como la dislipidemia, la edad y la genética, llevan a daño celular directo e indirecto (daño de los vasa nervorum) que finaliza con el daño de la fibra nerviosa ${ }^{(9)}$.

Dentro de los elementos implicados está la vía de los polioles, con un aumento de la función de la aldolasa-reductasa, lo cual genera un aumento del sorbitol y de la fructosa que, a su vez, disminuye la disponibilidad de nicotinamida adenina dinucleótido fosfato (NADPH); esto lleva a una mala regulación de los radicales libres de oxígeno. Otro elemento implicado es la vía de la hexosamina, que aumenta a glicosilación con la formación de productos de glicación avanzada terminales, que contribuyen al aumento de los niveles de angiotensina y proteína quinasa $\mathrm{C}$, los cuales generan vasoconstricción y disfunción endotelial subsiguiente. Por último, está la activación anormal de la proteína quinasa $\mathrm{C}$ con la síntesis de diacilglicerol secundaria a la hiperglucemia, que genera más vasoconstricción y daño isquémico en el tejido nervioso ${ }^{(10,11)}$.

Todo lo anterior termina en la degeneración neuronal y axonal, pérdida de fibras nerviosas, desmielinización y alteración en la regeneración nerviosa. El daño axonal tiene predominio inicial por las fibras nerviosas largas. Es por esto por lo que el primer sistema comprometido es el parasimpático, ya que el vago es el nervio autónomo más largo que existe y es el responsable del 75 \% de la función parasimpática. Esta alteración en el sistema vagal genera un aumento en la función simpática por la pérdida de la contrarregulación ${ }^{(12)}$. A medida que avanza la enfermedad, hay también una denervación simpática, tanto periférica como a nivel cardíaco, que se presenta del ápex hacia la base ${ }^{(12)}$ (Figura 1).
Figura 1. Fisiopatología de la neuropatía autonómica cardíaca.

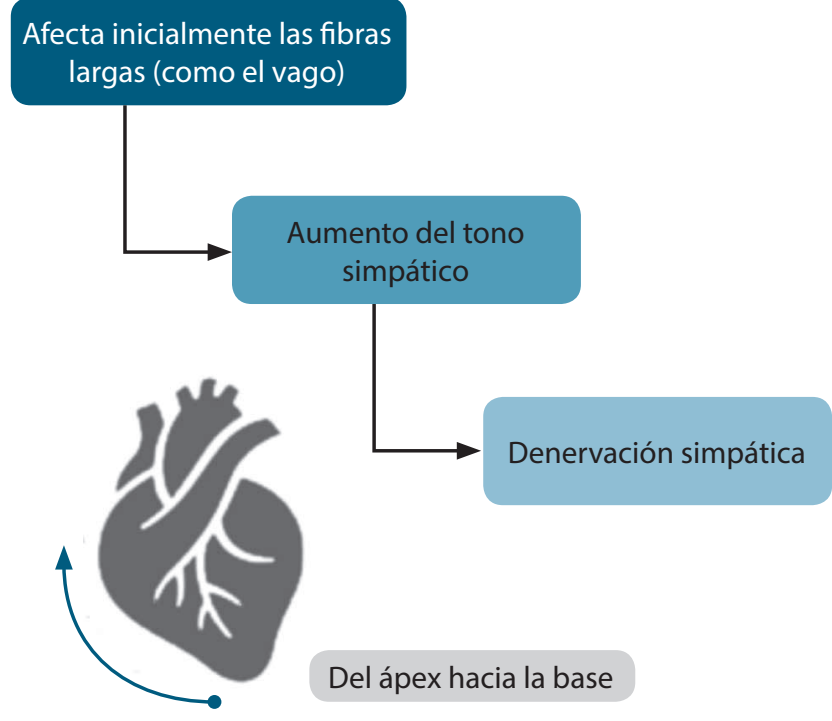

\section{Manifestaciones clínicas}

Son muchos los sistemas que se pueden ver afectados por la neuropatía autonómica de la diabetes, entre ellos se encuentran: el sistema gastrointestinal (dismotilidad esofágica, gastroparesia, diarrea o constipación, e incontinencia fecal), el sistema genitourinario (disfunción eréctil, eyaculación retrógrada, vejiga neurogénica y disfunción sexual femenina), el sistema sudomotor (anhidrosis, hiperhidrosis, intolerancia al calor, piel seca, alteraciones en termorregulación y edemas), la función pupilar (alteración de la función pupilomotora, pupilas pseudo ArgyllRobertson) y, por último y más relevante en esta revisión, el sistema cardiovascular con la CAN $^{(12)}$ (Figura 2).

A nivel cardiovascular, el espectro de las alteraciones varía desde el aumento de la frecuencia cardíaca en reposo hasta la muerte súbita. El signo clínico más temprano y común es la taquicardia en reposo, la cual se da por una activación del sistema simpático sin oposición del parasimpático, lo que refleja la alteración vagal inicial ${ }^{(10)}$. A medida que avanza la enfermedad, los pacientes presentan intolerancia al ejercicio por incompetencia cronotrópica, con alteración en el aumento del gasto cardíaco (GC) por modulación simpática errónea y, finalmente, pueden terminar con una frecuencia cardíaca fija, que es indicativo de la denervación cardíaca total y puede llevar a infarto agudo de miocardio silente y muerte ${ }^{(12)}$.

Además, pueden presentarse con alteración del patrón circadiano de la presión arterial (patrón no dipping en la monitorización ambulatoria de la presión arterial) e hipotensión ortostática de predominio diurno. Esto refleja una combinación entre las alteraciones centrales y periféricas del sistema simpático, lo que genera alteraciones en la calidad de vida por caídas y síncope ${ }^{(7)}$ (Figuras 3 y 4). 
Figura 2. Manifestaciones de la neuropatía autonómica diabética.

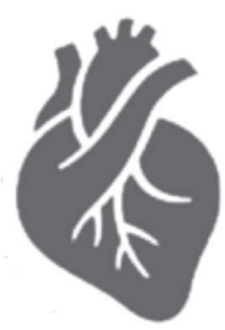

Taquicardia - bradicardia

Disfunción ventricular

Poca tolerancia al ejercicio

Hipotensión ortostática con

hipertensión nocturna

Inestabilidad cardiovascular durante

procedimientos quirúrgicos

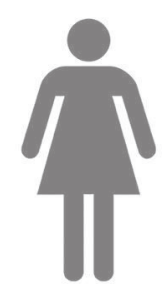

Anhidrosis e hiperhidrosis

Intolerancia al calor

Piel seca, alteraciones en la termorregulación

Edemas

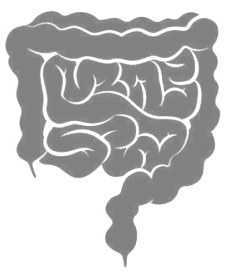

Dismotilidad esofágica

Gastroparesia

Diarrea - constipación

Incontinencia fecal

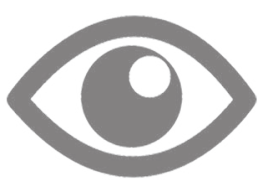

Alteración de la función

pupilomotora

pseudo Argyll-Robertson

Disfunción eréctil

Eyaculación retrógrada

Vejiga neurogénica

Disfunción sexual femenina
ㅁㅁㅁ

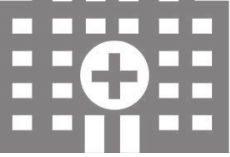

Hipoglucemia no sintomática SAHOS

Depresión y ansiedad

SAHOS: síndrome de apnea e hipopnea obstructiva del sueño.

Figura 3. Manifestaciones cardiovasculares de la neuropatía autonómica diabética.

\section{Taquicardia en reposo}

Marcador temprano. Se da por una activación del sistema simpático sin oposición del parasimpático.

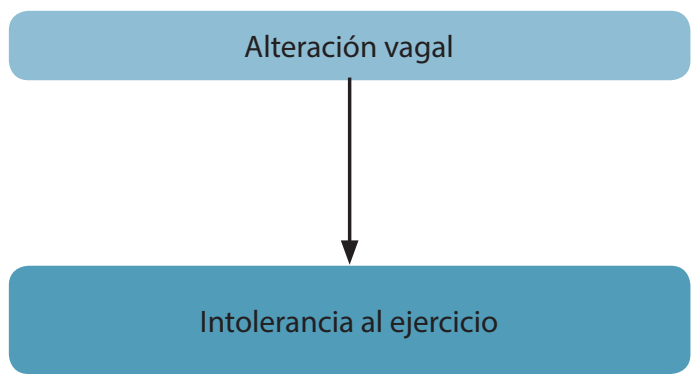

Incompetencia cronotrópica

\section{FC fija}

Enfermedad avanzada

Denervación cardíaca: FC 80-90 fija, IAM sin dolor y muerte súbita

Alteración en el aumento del GC por modulación simpática alterada. Hay taquicardia, pero sin variación con la actividad.

FC: frecuencia cardíaca; GC: gasto cardíaco; IAM: infarto agudo de miocardio. 
Figura 4. Manifestaciones cardiovasculares de la neuropatía autonómica diabética.

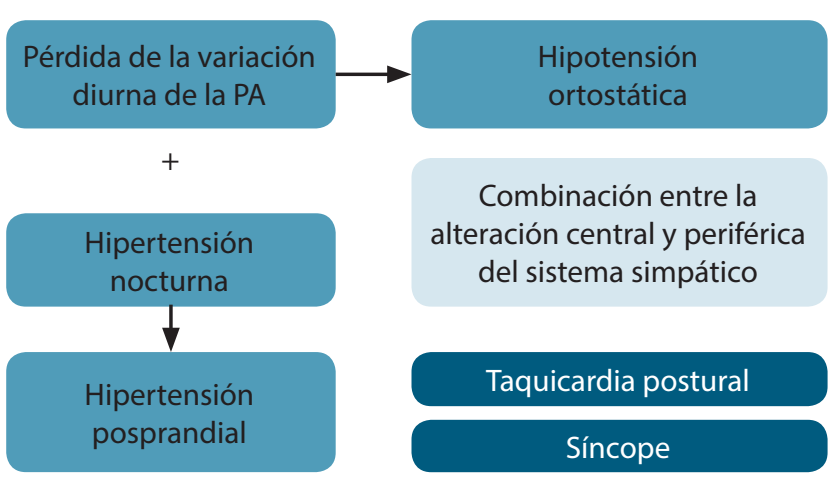

PA: presión arterial.

Otra de las manifestaciones clínicas más relevantes es la inestabilidad intraoperatoria, con una mortalidad 2-3 veces mayor en diabéticos con CAN que sin CAN llevados a cirugía. En estos pacientes la respuesta autonómica no logra compensar los efectos vasodilatadores y cronotrópicos negativos de la anestesia, lo que lleva a una hipotermia intraoperatoria más severa, desaceleración del metabolismo de los medicamentos anestésicos y mayor profundidad y demora en la recuperación ${ }^{(7)}$. La prolongación del intervalo QT no es infrecuente y se da por la heterogeneidad de las propiedades eléctricas del miocardio; hay anomalías del complejo QRS con respecto a la onda T (vectocardiografía), dispersión en los períodos refractarios y las velocidades de conducción, siendo la prolongación un predictor independiente de muerte ${ }^{(13)}$.

\section{Diagnóstico}

En cuanto al diagnóstico, no hay una prueba de oro que pueda ser comparada con otros métodos. El abordaje se basa en la realización de varias pruebas que evalúen diferentes espectros de la función autonómica hasta llegar a: un diagnóstico probable (1 prueba alterada), confirmado ( 2 pruebas alteradas) o confirmado grave ( 2 pruebas alteradas más hipotensión ortostática) ${ }^{(12)}$. En la literatura se ha propuesto el uso de medicina nuclear con análogos radiomarcados de norepinefrina (gammagrafía con 123I-metaiodobenzylguanidina u 11C-hidroxiefedrina) para el diagnóstico de la enfermedad. No obstante, en la práctica clínica este método presenta una utilidad limitada por el alto costo y la pobre estandarización ${ }^{(12,14)}$.

El marcador más temprano hasta ahora estudiado es la disminución en la variabilidad de la frecuencia cardíaca, que se refiere a las fluctuaciones del intervalo RR por la modulación autonómica. Estas fluctuaciones son evaluadas frecuentemente mediante índices como el standard deviation of all normal $R$ - $R$ intervals (SDNN) o el root-mean-square of the difference of successive $R-R$ interval (rMSSD) en la monitorización Holter de 24 horas $^{(15)}$.

Según la Asociación Americana de Diabetes (ADA), el cribado de esta entidad en pacientes diabéticos debería realizarse en tres escenarios ${ }^{(16)}$ :

1. Aquellos con complicaciones microvasculares o neuropáticas ya establecidas y conocidas.

2. Ante signos o síntomas de neuropatía cardíaca, excluyendo primero otras comorbilidades o efectos/interacciones medicamentosas como causantes.

3. Pacientes con hipoglucemias inadvertidas.

Es así como cualquiera de los tres grupos mencionados debería ser sometido a una serie de pruebas para establecer o excluir el diagnóstico de CAN. Las pruebas más aceptadas actualmente se enuncian en la Tabla $\mathbf{1}^{(12)}$.

Tabla 1. Pruebas de tamizaje más usadas para el diagnóstico de neuropatía autonómica diabética cardíaca

Método de

tamización

Frecuencia cardíaca en reposo

Variación de la frecuencia cardíaca latido a latido

Respuesta de la frecuencia cardíaca con la bipedestación

Respuesta de

la frecuencia cardíaca con maniobra de Valsalva

Respuesta de la presión arterial sistólica con la bipedestación

Repuesta diastólica de la presión al ejercicio isométrico

Intervalo QT/QTc en el electrocardiograma

Flujo neurovascular
Hallazgos anormales

Frecuencia cardíaca >100 latidos/minuto

Paciente en supino con frecuencia respiratoria de 6 respiraciones/minuto, monitorización electrocardiográfica de la frecuencia cardíaca, variabilidad de frecuencia $<10$ latidos, relación E/I de intervalo $R R>1,17$.

Durante la monitorización electrocardiográfica continua se mide el intervalo RR en el latido 15 y 30 después de la bipedestación. Razón $<1,03$.

Espiración forzada en un manómetro a $40 \mathrm{~mm} \mathrm{Hg}$ durante 15 segundos mientras se realiza el electrocardiograma. Presencia de bradicardia y vasodilatación periférica en el esfuerzo y taquicardia con hipotensión al detener la maniobra. Razón de intervalo $\mathrm{RR}<1,2$.

Medición de la presión arterial sistólica en supino, luego en bipedestación después de 2 minutos. Presencia de una caída de presión arterial $>30 \mathrm{~mm} \mathrm{Hg}$.

Prensión de un dinamómetro para establecer un máximo. Luego, con el brazo contralateral se prensa $30 \%$ del máximo por 5 minutos. Aumento de la presión diastólica $<16 \mathrm{~mm} \mathrm{Hg}$.

QTc $>440 \mathrm{~ms}$

Medición de la respuesta periférica simpática de nocicepción mediante ecografía Doppler. 


\section{Diagnóstico diferencial}

Dentro del enfoque de la enfermedad se deben tener en cuenta algunos diagnósticos diferenciales antes de poder atribuirle la sintomatología del paciente a esta entidad, esto sin llegar a ser un diagnóstico de exclusión. Entre las enfermedades más comunes que se deben descartar: los trastornos metabólicos asociados con alteraciones tiroideas y renales; enfermedades sistémicas, como paraproteinemias y vasculitis; posibles infecciones, como VIH, hepatitis B o C; trastornos inflamatorios como la radiculopatía; y las enfermedades crónicas desmielinizantes (CIDP). Siempre se deben descartar deficiencias nutricionales a través de la medición sérica de vitaminas (en especial la B12) y, por último, buscar la relación de los síntomas con el consumo de medicamentos como amiodarona, colchicina o dapsona. La historia clínica y el tiempo de evolución de la enfermedad pueden orientar en el diagnóstico ${ }^{(6)}$.

\section{Tratamiento}

En cuanto a las generalidades del manejo, es fundamental la prevención de los factores modificables que impactan en los desenlaces de daño, como la hiperglucemia, tabaquismo, hipertensión e hiperlipidemia ${ }^{(17)}$. En cuanto a la hiperglucemia, se ha publicado evidencia contradictoria a través del tiempo. En la diabetes mellitus tipo 1, los estudios DCCT ${ }^{(8)}$ y EDIC ${ }^{(18)}$ demostraron que la terapia intensiva glucémica reduce la incidencia de CAN frente a la terapia convencional en 5 años de seguimiento (14\% frente a $7 \% ; p<0,004)$, y que a largo plazo $^{(19,20)}$ tiene una tasa significativamente menor. Por el contrario, la evidencia no ha sido tan firme en la diabetes mellitus tipo 2; el estudio Anglo-Danish-Dutch Study demostró que no había diferencia en los grupos a 6 años de seguimiento, pero el Steno 2 Trial confirmó que un control multidisciplinario, teniendo en cuenta la glucemia, el perfil lipídico, la presión arterial y la proteinuria, tiene impacto en la mejoría de la CAN a largo plazo ${ }^{(12)}$.

El tratamiento sintomático se divide en medidas farmacológicas y no farmacológicas. Las medidas no farmacológicas son la piedra angular para evitar el empeoramiento marcado del ortostatismo; entre ellas se encuentran: realizar cambios posturales progresivos, tensión y estiramiento de piernas antes de la bipedestación, dorsiflexión de pies y ejercicio isométrico de agarre con las manos. Si hay mejoría parcial realizando estas medidas, tienen cabida las medidas farmacológicas con el uso de medicamentos que aumentan el volumen plasmático, como la fludrocortisona, sumado al consumo en dietas ricas en sal (21). Otros medicamentos que se han propuesto para el tratamiento sintomático son los agonistas $\alpha 1$, la midrodina, la doxidopa (la cual es una prodroga de la norepinefrina) y el octreótido (análogo de la somatostatina en enfermedades muy avanzadas y resistentes a otras medidas) ${ }^{(4)}$.

Los inhibidores del cotransportador sodio-glucosa tipo 2 (SGLT2) han demostrado múltiples efectos benéficos cardiovasculares en los pacientes con diabetes mellitus tipo $2^{(1)}$. En un estudio realizado por Ferdinand y colaboradores ${ }^{(22)}$ se demostró que la empagliflozina, además de lograr una reducción en el peso del paciente, tiene impacto en la disminución de la presión arterial media de hasta 8,3 mm Hg, siendo mayor el efecto a mayor tiempo de observación; esto sugiere que los efectos del medicamento alcanzan su máxima capacidad después de 6 meses del inicio. Así mismo, en el estudio DECLARE-TIMI58 ${ }^{(23)}$ se demostró que la dapagliflozina tiene seguridad cardiovascular.

La reducción de la presión arterial con estos medicamentos se da sin generar taquicardia refleja. Es por esta razón que se ha planteado la posibilidad de un papel en la modulación de la actividad nerviosa autónoma, ejerciendo un efecto simpático-inhibitorio $^{(22)}$. Lo anterior se ha explicado por los vínculos e interacciones encontradas entre la actividad nerviosa simpática y el síndrome metabólico, obesidad, HTA y DM. Se han realizado estudios en murinos con HTA, DM y obesidad, que han demostrado que el tratamiento con SGLT2, además de disminuir los valores de presión arterial, normaliza el patrón circadiano de la PA sin cambios en la FC, proponiendo efectos benéficos en la modulación del sistema nervioso autónomo por un mecanismo aún no conocido ${ }^{(24)}$.

\section{Conclusión}

La CAN debe ser reconocida como una complicación prevalente e importante por los médicos que se vean enfrentados a pacientes con diabetes mellitus y cualquiera de sus complicaciones asociadas. Se debe buscar activamente esta entidad para evitar su aparición o mitigar sus efectos deletéreos, ya sea instaurando un control glucémico más estricto (evitando la hipoglucemia) o tratamientos específicos, teniendo en cuenta los SGLT2 como terapia prometedora; y, más importante aún, reconociendo esta entidad dentro de las metas establecidas para el adecuado manejo de los pacientes diabéticos.

\section{Conflictos de interés}

Los autores declaran no tener ningún conflicto de interés para esta revisión. 


\section{Referencias}

1. Association AD. Standards of Medical Care in Diabetes-2019 Abridged for Primary Care Providers. Clin Diabetes. 2019;37(1):11-34.

2. Codinach Huix P, Freixa Pamias R. Miocardiopatía diabética: concepto, función cardiaca y patogenia. An Med Interna. 2002;19(6):55-62.

3. Vinik AI, Maser RE, Mitchell BD, Freeman R. Diabetic autonomic neuropathy. Diabetes Care. 2003;26(5):1553-79.

4. Vinik AI, Casellini C, Parson HK, Colberg SR, Nevoret ML. Cardiac autonomic neuropathy in diabetes: A predictor of cardiometabolic events. Front Neurosci. 2018;12(AUG):1-11

5. Rathmann W, Ziegler D, Jahnke M, Haastert B, Gries FA. Mortality in diabetic patients with cardiovascular autonomic neuropathy. Diabet Med J Br Diabet Assoc. 1993;10(9):820-4

6. Serhiyenko VA, Serhiyenko AA. Cardiac autonomic neuropathy: Risk factors, diagnosis and treatment. World J Diabetes. 2018;9(1):1-24.

7. Balcıoğlu AS, Müderrisoğlu H. Diabetes and cardiac autonomic neuropathy: Clinical manifestations, cardiovascular consequences, diagnosis and treatment. World J Diabetes. 2015;6(1):80-91.

8. The effect of intensive diabetes therapy on the development and progression of neuropathy. The Diabetes Control and Complications Trial Research Group. Ann Intern Med. 1995;122(8):561-8.

9. Pop-Busui R. Cardiac autonomic neuropathy in diabetes: a clinical perspective. Diabetes Care. 2010;33(2):434-41.

10. Brownlee M. The pathobiology of diabetic complications: a unifying mechanism. Diabetes. 2005;54(6):1615-25.

11. Oakley I, Emond L. Diabetic cardiac autonomic neuropathy and anesthetic management: review of the literature. AANA J. 2011;79(6):473-9.

12. Verrotti A, Prezioso G, Scattoni R, Chiarelli F. Autonomic Neuropathy in Diabetes Mellitus. Front Endocrinol [Internet]. [citado 8 de octubre de 2019];5. Disponible en: https://www.ncbi.nlm.nih.gov/pmc/articles/ PMC4249492/

13. Duque M, Díaz JC, Molina DI, Gómez E, Márquez G, López P, et al. Diabetic autonomic cardiovascular neuropathy. Rev Colomb Cardiol. 2013;20(2):80-7.

14. Raffel DM, Wieland DM. Assessment of cardiac sympathetic nerve integrity with positron emission tomography. Nucl Med Biol. 2001;28(5):541-59.
15. Heart rate variability. Standards of measurement, physiological interpretation, and clinical use. Task Force of the European Society of Cardiology and the North American Society of Pacing and Electrophysiology. Eur Heart J. 1996;17(3):354-81.

16. Pop-Busui R, Boulton AJM, Feldman EL, Bril V, Freeman R, Malik RA, et al Diabetic Neuropathy: A Position Statement by the American Diabetes Association. Diabetes Care. 2017;40(1):136-54

17. Gibbons CH, Freeman R, Tecilazich F, Dinh T, Lyons TE, Gnardellis C, et al The evolving natural history of neurophysiologic function in patients with well-controlled diabetes. J Peripher Nerv Syst JPNS. 2013;18(2):153-61.

18. Nathan DM, DCCT/EDIC Research Group. The diabetes control and complications trial/epidemiology of diabetes interventions and complications study at 30 years: overview. Diabetes Care. 2014:37(1):9-16.

19. Theilade S, Lajer M, Persson F, Joergensen C, Rossing P. Arterial stiffness is associated with cardiovascular, renal, retinal, and autonomic disease in type 1 diabetes. Diabetes Care. 2013;36(3):715-21.

20. Secrest AM, Marshall SL, Miller RG, Prince CT, Orchard TJ. Pulse wave analysis and cardiac autonomic neuropathy in type 1 diabetes: a report from the Pittsburgh Epidemiology of Diabetes Complications Study. Diabetes Technol Ther. 2011;13(12):1264-8.

21. Goswami N, Blaber AP, Hinghofer-Szalkay H, Montani J-P. Orthostatic Intolerance in Older Persons: Etiology and Countermeasures. Front Physiol [Internet]. de 2017 [citado 8 de octubre de 2019]. Disponible en: https:// www.ncbi.nlm.nih.gov/pmc/articles/PMC5677785/

22. Ferdinand KC, Izzo JL, Lee J, Meng L, George J, Salsali A, et al. Antihyperglycemic and Blood Pressure Effects of Empagliflozin in Black Patients With Type 2 Diabetes Mellitus and Hypertension. Circulation. 2019;139(18):2098-109.

23. Wiviott SD, Raz I, Bonaca MP, Mosenzon O, Kato ET, Cahn A, et al. Dapagliflozin and Cardiovascular Outcomes in Type 2 Diabetes. 2019;380(4):347-57.

24. Wan N, Rahman A, Hitomi H, Nishiyama A. The Effects of Sodium-Glucose Cotransporter 2 Inhibitors on Sympathetic Nervous Activity. Front Endocrinol. 2018;9:421. 